\title{
PERIPHERAL BLOOD EOSINOPHILS AS MARKER OF SPUTUM EOSINOPHILIA AND OUTCOME OF COPD EXACERBATION
}

(C) by Acta Medica Saliniana ISSN 0350-364X

Type of manuscript: Professional papers

\section{Title:}

PERIPHERAL BLOOD EOSINOPHILS AS MARKER OF SPUTUM EOSINOPHILIA AND OUTCOME OF COPD EXACERBATION

\section{Authors:}

Jusufovic Edin ${ }^{1,2}$, Kosnik Mitja ${ }^{3,4}$ Nurkic Jasmina ${ }^{1,5}$

Arifhodzic Nermina ${ }^{5}$

Al-Ahmad Mona ${ }^{6}$

Becarevic Munevera

Brekalo-Lazarevic Sanja ${ }^{1}$

Mosorovic Nehra ${ }^{1}$

\section{DOI: $10.5457 / 487$}

\section{Afiliations:}

${ }^{1}$ University in Tuzla, Medical Faculty, Tuzla, Bosnia and Herzegovina, ${ }^{2}$ Health and Educational Medical Centre, Centre for Pulmonary Diseases, Tuzla, Bosnia and Herzegovina, ${ }^{3}$ University in Ljubljana, Faculty of Medicine, Ljubljana, Slovenia, ${ }^{4}$ The University Clinic of Pulmonary and Allergic Diseases, Clinic of Respiratory and Allergic Diseases, Golnik, Slovenia,

${ }^{5}$ Immunology Unit, Allergy Department, Al-Rashed Allergy Center, Kuwait, ${ }^{6}$ Microbiology Department, Faculty of Medicine, Kuwait University

Received:

11.01.2019.

Corresponding author:

Jusufovic Edin

Email: edin.jusufovic@bih.net.ba

Sputum eosinophils might predict response to inhaled corticosteroids (ICS) in patients with advanced chronic obstructive pulmonary disease (COPD). Induction of sputum requires expertise and may not always be successful.

Aim was to investigate correlation and predictive relationship between peripheral blood eosinophils (bEo) and sputum eosinophils (sEo), and impact of peripheral blood eosinophilia on outcome of COPD exacerbation.

120 current smokers with COPD (GOLD group C) $(57.4 \pm 0.92$ years, M/F ratio 1.4), with no blood $\left(\geq 7 \%\right.$ or $\left.>0.43 \times 10^{\%} / \mathrm{L}\right)$ nor sputum $(\geq 3 \%)$ eosinophilia, were treated with moderate dose of ICS and long-acting bronchodilatator during stable disease, but systemic corticosteroids and antibiotics during exacerbation. According to sputum eosinophilia $(\geq 4 \%)$ during exacerbation, patients were divided into eosinophilic $(n=45)$ and noneosinophilic group $(n=75)$.

In stable disease, bEo and sEo were similar in both groups ( $p>0.05$ ). During exacerbation, bEo and sEo were significantly higher in eosinophilic group (eosinophilic vs. noneosinophilic: blood: $1.42 \pm 0.39 \times 10^{9} / \mathrm{l}$ vs. $0.23 \pm 0.02 \times 10^{9} / \mathrm{l}$, p $<0.001$; sputum: $8 \%(4,19)$ vs. $1 \%(0,3), p<0.0001$ ), but bEo correlated with $s$ Eo in both groups (eosinophilic: $r=0.52$, $p<0.001$; non-eosinophilic: $r=0.25, p<0.05$ ). Relative bEo predicted sputum eosinophilia (area under the curve $=0.71$, standard error $=0.05 ; 95 \%$ confidence interval $[\mathrm{Cl}]=0.61-0.81$; $\mathrm{p}<0.001$ ) and enabled identification of the presence or absence of sputum eosinophilia in $82 \%$ of the cases at a threshold of $\geq 4 \%$ (specificity $=83.56 \%$, sensitivity $=93.83 \%$, positive likelihood ratio=3.67).

Eosinophilic group during exacerbation showed less frequent hospitalisations and shorter exacerbation (eosinophilic vs. non-eosinophilic: hospitalisations: $26.7 \%$ vs. $60.0 \%, p<0.001$; duration of exacerbation (days): $8.1 \pm 0.35$ vs. $10.13 \pm 0.31, p<0.0001$ ).

In COPD exacerbation, relative peripheral blood eosinophils $\geq 4 \%$ might identify sputum eosinophilia. Blood eosinophilia indicate better outcome of COPD exacerbation. Further investigations are needed to predict eosinophilic exacerbation in COPD patients, with prior absence of sputum or blood eosinophilia.

Key words: chronic obstructive pulmonary disease, eosinophils, blood, sputum.

\section{INTRODUCTION}

Besides asthma, airway eosinophilia is now well recognized inflammatory pattern in chronic obstructive pulmonary disease (COPD) (1). There is increasing body of evidence that the eosinophils might play an important role in $10-40 \%$ of patients with COPD (2). A number of recent studies have shown that blood eosinophil counts could predict the magnitude of the effect of inhaled corticosteroids (ICS), added on top of regular maintenance bronchodilator treatment in preventing COPD exacerbations $(3,4)$. A recent study examining ICS withdrawal on a background of dual bronchodilator therapy demonstrated that both FEV1 loss and an increase in exacerbation frequency associated with ICS withdrawal was greatest among patients with a blood eosinophil count $\geq 300$ cells $/ \mu$ at baseline (5). Eosinophilic COPD, defined as sputum eosinophils 3\%, is reported during acute exacerbations in up to $28 \%$ of cases (6). In the same time, although airway eosinophilia has been found in COPD during exacerbations, an increase in airway eosinophils in patients with stable conditions has been found in some studies, but not in others (7). Interestingly, in periods of COPD stability, it is found in approximately $34 \%$ (8) to $38 \%$ (9) of patients. Airway eosinophilia is a reliable predictor of responsiveness to inhaled and oral corticosteroid therapies in COPD $(9,10)$. The detection and measurement 
of airway eosinophilia mostly require the assessment of induced sputum (1). Although sputum induction is considered a direct and reliable method of assessing airway inflammation, it has a number of limitations. In addition to being unsuitable for point-of-care testing, it requires expertise and may not be always successful (failure rate of up to $30 \%)(11,12)$. Due to these reasons, the search for minimally invasive and easily applicable diagnostic tools that can predict sputum eosinophilia in COPD has intensified $(6,10,11)$. The use of peripheral blood eosinophils (bEo) counts as a potential alternative is attracting profound interest owing to its ease of application in clinical practice (10). However, little is known about measurement stability, which is important for understanding the utility of bEo as a potential biomarker (13). In asthma patients the predictive value of blood eosinophils in detecting sputum eosinophils (sEo) count asthma has been reported (14). However, in COPD patients, only few studies have addressed this, demonstrating the association between sEo and bEo counts $(6,15)$, as well as potential ability of blood eosinophils to serve as a marker of response to corticosteroid treatments during exacerbation (6). On the other side, increment of research studied clinical COPD features and bEo resulted in introducing the bEo count as a biomarker for estimating the efficacy of inhaled corticosteroids for the prevention of COPD exacerbations (16). Nevertheless, studies examining the utility of bEo in detecting sEo in COPD patients are still lacking, both in stable disease (10), as well as during exacerbation. On the other side, the results of the published studies are contradictory and the differences between studies may relate to differences in methodology, including the use of background long acting bronchodilator medication(s) which may minimize any effect of ICS withdrawal (16).

Aim of our study was to investigate the correlation and predictive relationship between bEo to sputum eosinophils sEo, as well as the impact of peripheral blood eosinophilia on outcome of COPD exacerbations.

\section{SUBJECTS AND METHODS}

Study design: A prospective, 12 months lasting study was conducted from May 2016 to May 2017 year in Centre for Pulmonary Diseases of Public Medical and Educational Institution Tuzla, Bosnia and Herzegovina.

Subjects: 120 current smokers COPD patients with GOLD group C ( $\geq 2$ exacerbations during observational year with mMRC score $\leq 1$ and CAT score $<10$ ) with no blood $\left(\geq 7 \%\right.$ or $\left.>0.43 \times 10^{9} / \mathrm{L}\right)$ nor sputum $(\geq 3 \%)$ eosinophilia during stable disease. Blood eosinophilia was defined as Eo values During stable disease all patients were treated with moderate dose of ICS and long-acting bronchodilatator $(250 \mathrm{mcg}$ fluticasone propionate +100 mcg salmeterol HFA, per day) with inhaled salbutamol as needed. According to sputum eosinophilia $(\geq 4 \%)$ during the next exacerbation, patients were divided into eosinophilic $(n=45)$ and non-eosinophilic group $(\mathrm{n}=75)$.
Adherence and compliance to inhaled therapy were checked in all patients before enrolment to study. Inclusion criteria were: 1. COPD, GOLD C group, 2. good adherence and compliance to inhaled therapy. Exclusion criteria were: 1 . asthma-COPD overlap (ACO), 2. actual pneumonia or acute infections excluded by chest $X$ ray and $C$ reactive protein, 3 . bronchiectasis, 4 . active tuberculosis, 5. helmintiasis, 6. allergy (total IgE $\geq 100$ $\mathrm{IU} / \mathrm{ml}$ or positive skin prick testing on standard inhaled and nutritive allergens), 6. congestive heart failure.

sEo and bEo were measured and compared between during stable disease and one egzacerbation. According sputum eosinophilia $(\geq 4 \%)$ during exacerbation, patients were divided into eosinophilic $(n=45)$ and non-eosinophilic group $(n=75)$. Also, predicted forced expiratory volume in first second (FEV1\%), modified Medical Research Council (mMRC) score and COPD Assessment Test (CAT) score were done in all patients during stable disease.

Sputum induction and processing: Sputum was conducted concurrently with the inhaled hypertonic (3\%) saline solution. Subjects were asked to rinse their mouth with water before the procedure to help eliminate squamous cell contamination of the sputum sample. They were asked to cough between each dose of nebulised saline to clear their throat and expectorate into a plastic Petri dish. This procedure continued until an adequate sample containing $>0.5$ $\mathrm{ml}$ visible mucocellular material was obtained. After induction, sputum was treated by adding four volumes of $0.1 \%$ dithiothreitol and rotated for 30 minutes at $37^{\circ} \mathrm{C}$, followed by four volumes of phosphate buffered saline. The suspension was filtered through $60 \mu \mathrm{m}$ nylon gauze and then centrifuged at $200 \mathrm{~g}$ for 10 minutes. Supernatant was aspirated and $70 \mu \mathrm{l}$ placed for slide preparation. The quality of induced sputum samples was assessed based on the presence of an adequate number of cells for enumeration, the presence of pulmonary macrophages on the slide, and the proportion of squamous epithelial cells. This gave a quality score ranging from 0 (poor quality) to 6 (good quality sample). A differential cell count was obtained by counting 400 non-squamous cells on slides fixed with methanol and stained with May Grunwald Giemsa. Eosinophils were enumerated as the percentage of 400 cells on slides fixed with methanol and stained with Chromotrope 2R. Metachromatic cells were counted as the percentage of 1500 cells on slides fixed in Carnoy's solution and stained with acidic toluidine blue.

Peripheral blood relative eosinophil count was determined by an automated hematology analyzer (Cell-Dyn 4000; Abbott Laboratories, Abbott Park, IL).

Spirometric tests: Subjects withheld salbutamol $400 \mu \mathrm{g}$ for 20 minutes before testing. Height and weight were recorded before performing three reproducible forced expiratory manoeuvres wearing nose clips to measure forced expiratory volume in 1 second (FEV1) and vital capacity (VC).

mMRC scale is a self-rating tool to measure the degree of disability that breathlessness poses on day-to-day 
activities on a scale from 0 to 4 : 0 , no breathlessness except on strenuous exercise; 1 , shortness of breath when hurrying on the level or walking up a slight hill; 2 , walks slower than people of same age on the level because of breathlessness or has to stop to catch breath when walking at their own pace on the level; 3, stops for breath after walking $\sim 100 \mathrm{~m}$ or after few minutes on the level; and 4, too breathless to leave the house, or breathless when dressing or undressing (17).

CAT, that been developed to provide a simple and reliable measure of health status in COPD patients, consists of eight items (cough, phlegm, chest tightness, breathlessness, limited activities, confidence leaving home, sleeplessness and energy) defined with contrasting adjectives. Item scores range from 0 to 5 points resulting in a CAT total score ranging from 0 to 40 points. A CAT total score of $\geq 10$ points or $\geq 18$ points has been suggested to classify patients as highly symptomatic. The minimal clinically important difference of the CAT is 2 points (18).

Need for hospitalisation was defined on the presence of the risk factors associated with a complicated exacerbation: 1. marked increase in intensity of symptoms, 2 . FEV $1<50 \%$ predicted, 3 . $\geq 4$ exacerbations in the last year, 4. presence of serious comorbidities (ischemic heart disease, ...), 5. use of home oxygen, 6. chronic oral steroid use, 7. older age and 8. failure of an exacerbation to respond to initial medical management. If a least one risk factor was present at the moment of admission, COPD exacerbation was considered as a complex acute exacerbation with need for hospitalisation $(16,19)$.

During exacerbation all patients were treated according GOLD recommendations with: 1 . supplemental oxygen (with target saturation of 88-92\%), 2. short-acting inhaled beta2-agonists with or without short-acting anticholinergics, $3.40 \mathrm{mg}$ prednisone or hydrocortisone $100 \mathrm{mg}$ per day for 5 to 10 days and 4 . antibiotics (azitromicin per os or cefazolin intravenous) during 5 to 10 days.

\section{Statistical analysis}

Non-parametric and parametric methods were used to calculate statistical significance. KolmogorovSmirnov test and Shapiro-Wilk normality test were used in order to test the normality of distribution of variables. Mean values were shown as arithmetic mean \pm standard deviation in case of normal distribution of variables (age, BMI, FEV1 (\%), bEo and duration of hospitalisation), or median [minimum value, maximum value] in case of non-normal distribution (sEo, mMRC score, CAT score and number of exacerbations in last year). Two-sided student's t-test, Mann-Whitney test, Fisher's test and $\chi 2$ test were used for calculating the difference between the groups. Spearman correlation was used to analyze the relationship between bEo and sEo. The ROC area under the curve was used to assess the degree of sensitivity and specificity at a cut off point. The statistical hypotheses were tested at the level of $\alpha=0.05$, and the difference between the groups in the sample was considered significant when $\mathrm{p}<0.05$ according to Bonferroni correction for 5 tests. Statistical significance was depicted as: $p<0.05, p<0.01$ and $\mathrm{p}<0.001$. All data were analysed using GraphPad Prism version 7 (San Diego, California, USA).

\section{RESULTS}

120 current smokers $(57.4 \pm 0.92$ years, $\mathrm{M} / \mathrm{F}$ ratio 1.4$)$ with COPD (GOLD group C) with no sputum nor blood eosinophilia during stable disease were included.

Distribution in age, gender, BMI, as well as in FEV1 \% predicted, mMRC score, CAT score, sEo and bEo during stable disease, were similar between eosinophilic and non-eosinophilic groups (Table I).

Table 1. Basic and follow up characteristics of eosinophilic and non-eosinophilic group

\begin{tabular}{|l|l|l|l|}
\hline Parameter & $\begin{array}{l}\text { Eosinophilic group } \\
(\mathrm{n}=45)\end{array}$ & $\begin{array}{l}\text { Non-eosinophilic group } \\
(\mathrm{n}=75)\end{array}$ & $p$ value \\
\hline Mean age (years) & $57.38 \pm 10.17$ & $57.47 \pm 10.01$ & 0.9628 \\
\hline Females (n; \%) & $21 ; 46.67 \%$ & $28 ; 37.33 \%$ & 0.3421 \\
\hline BMI (kg/m2) & $24.48 \pm 0.98$ & $24.11 \pm 2.47$ & 0.3436 \\
\hline FEV1 (\% predicted) & $51.39 \pm 18.86$ & $57.14 \pm 18.47$ & 0.1038 \\
\hline mMRC score & $0[0,1]$ & $0[0,1]$ & 0.4502 \\
\hline CAT score & $6[0,9]$ & $5[1,9]$ & 0.0885 \\
\hline sEo in stable disease (\%) & $1[0,2]$ & $2[0,2]$ & 0.1363 \\
\hline sEo in egzacerbation (\%) & $8[4,19]$ & $1[0,3]$ & $<0.0001^{*}$ \\
\hline$p$ value & $<0.0001^{*}$ & 0.9999 & \\
\hline bEo in stable disease (109/L) & $0.18 \pm 0.16$ & $0.21 \pm 0.19$ & 0.4123 \\
\hline bEo in egzacerbation (109/L) & $1.42 \pm 0.39$ & $0.23 \pm 0.02$ & $0.0002^{*}$ \\
\hline$p$ value & $0.0011^{*}$ & 0.0864 & \\
\hline Exacerbations in last year (n) & $4[2,6]$ & $3[2,4]$ & $0.0008^{*}$ \\
\hline Necessity for hospitalisation & $12(26.7 \%)$ & $45(60.0 \%)$ & $0.0006^{*}$ \\
\hline Duration of hospitalisation (days) & $8.09 \pm 2.38$ & $10.13 \pm 2.68$ & $<0.0001^{*}$ \\
\hline
\end{tabular}

$\left.{ }^{*}\right)$ difference was significant 
During exacerbation, eosinophilic group showed higher sEo and bEo in comparison to the values in stable disease, as well as to corresponding values in noneosinophilic group, where sEo and bEo were similar during exacerbation and stable disease (Table 1).
In addition, eosinophilic group showed higher number of COPD exacerbation during last year, but lower necessity for hospitalisation during exacerbation and lower duration of hospitalisation in comparison to noneosinophilic group (Table 1).

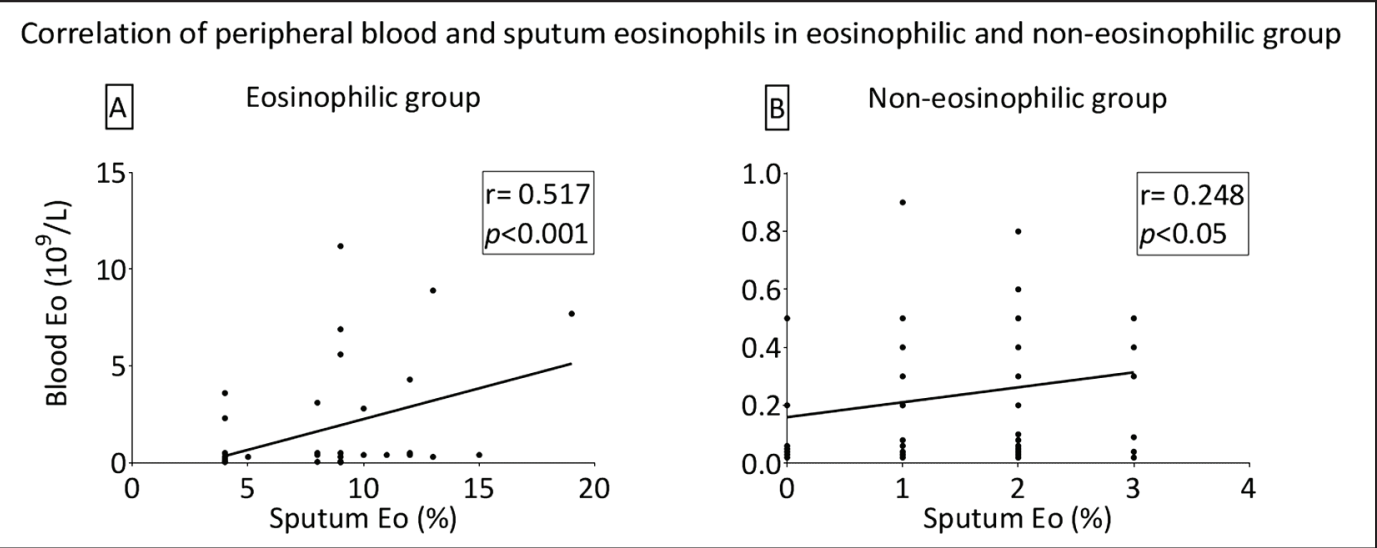

Graph 1. Shows correlation between peripheral blood and sputum eosinophils in eosinophilic and non-eosinophilic group

PBE correlated with SE in both eosinophilic and non-eosinophilic group (eosinophilic group: total Spearman coefficient 0.517 ; $95 \% \mathrm{CI}$ : 0.255 to 0.7082 ; $\mathrm{p}=0.0003$; non-eosinophilic group: total Spearman coefficient 0.248 ; 95\%CI: 0.01568 to 0.4553 ; $\mathrm{p}=0.0317$ ).

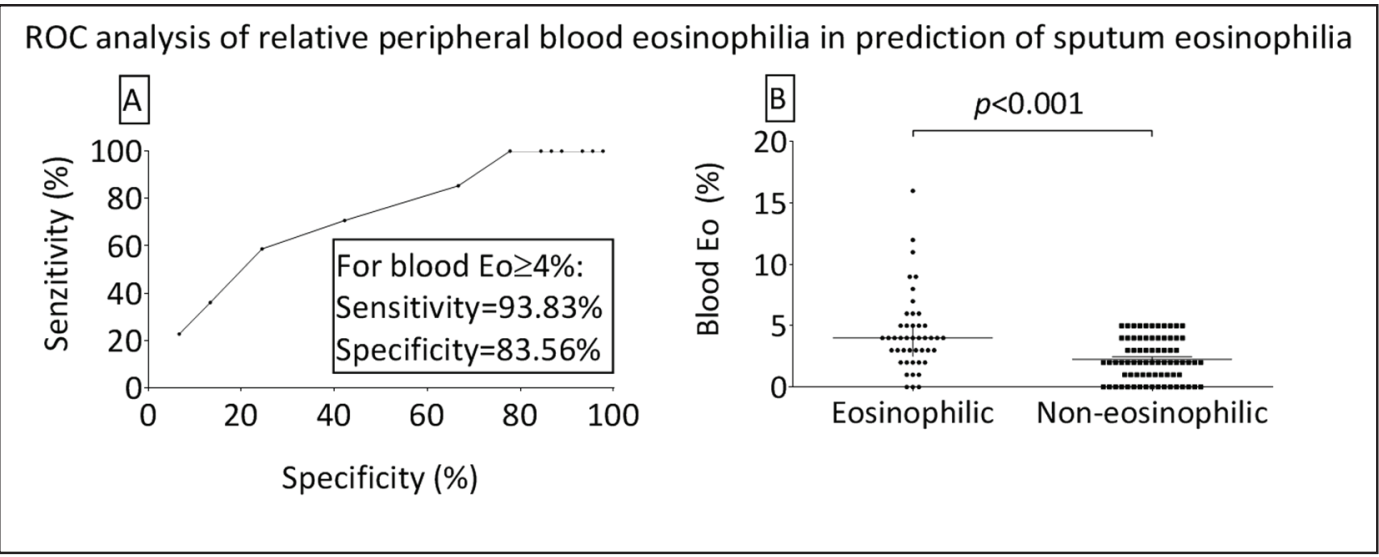

Graph 2 (A and B). Shows specificity and sensitivity of bEo to predict sputum eosinophilia (A), as well as bEo in eosinophilic and non-eosinophilic group (B)

Relative peripheral blood eosinophilia predicted sputum eosinophilia (area under the curve $=0.7105$, standard error $=0.04865 ; 95 \%$ confidence interval [CI] 0.6151 to $0.8059 ; \mathrm{p}=0.0001189$ ) and enabled identification of the presence or absence of sputum eosinophilia in $82 \%$ of the cases at a threshold of $\geq 4 \%$ (specificity $=83.56 \%$, sensitivity $=93.83 \%$, positive likelihood ratio=3.67).

\section{DISCUSSION}

Our study was performed on 120 COPD patients with GOLD group $\mathrm{C}$ in order to evaluate correlation and predictive relationship between bEo and sEo, as well as the impact of bEo on outcome of COPD exacerbations.
Most COPD exacerbations are associated with neutrophilic airways inflammation due to viral or bacterial infection, but increased sputum eosinophil counts may also be seen during exacerbations, especially in the presence of a viral infection (20). In the Copenhagen study, patients with COPD with higher bEo were more likely to have reported more infections and wheezing during colds, suggesting they may have had increased susceptibility to viral infection (21). The increased bEo count may reflect a past history of more frequent exacerbations with respiratory viruses (20) that are generally associated with more severe exacerbations and prolonged recovery (22). However, there is no evidence that the airway inflammatory profile is different between moderate and severe exacerbations, as currently defined clinically (20). In 
our study $37.5 \%$ of patients showed elevated sEo and bEo, but had no signs of acute infection. Furthermore, those patients showed more frequent exacerbations during last year than patients with low sEo or bEo (Table I). These indicate that a certain number of COPD patients could show an eosinophil elevation phenotype during exacerbations, which cannot be explained by infectious causes. Also other authors concluded that more information is needed on how sEo or bEo changed with time and how they were related to exacerbation triggers and their recovery (20). Further investigations of the COPD patients' subpopulation with elevated eosinophils only during exacerbations are warranted.

Several studies also investigated the predictive value of sEo and bEo on exacerbation risk and clinical response to $\operatorname{ICS}(6,23,24)$, as well as on pulmonary function $(25)$. It is described that bEo could predict exacerbation risk and the clinical response to ICS (6) and might represent an informative biomarker for exacerbation reduction with ICS/LABA in patients with COPD and a history of moderate/severe exacerbations (23). bEo could be used as a biomarker in severe COPD exacerbations for predicting higher readmission rates (24). Also, many other previous studies have consistently demonstrated that high bEo was associated with better response to ICS (21, 23, 25-29). Furthermore, baseline bEo $\geq 2 \%$ could identify a group of COPD patients with slower rates of decline in FEV1 when treated with ICS (25). bEo at screening were related to the exacerbation rate after complete ICS withdrawal in patients with severe to very severe COPD and a history of exacerbations and bEo of $4 \%$ or greater or 300 cells per $\mu \mathrm{L}$ or more might identify patients with a deleterious effect of ICS withdrawal (30). Also, higher bEo at admission for a COPD exacerbation was associated with increased COPD readmission rates in patients with infrequent COPD hospitalizations (31) and may be predictive of favourable response to therapy with steroids and bronchodilators (32). On the contrary, some studies have shown that the majority of COPD patients had circulating eosinophils $>2 \%$ and a significant association with the risk of severe COPD exacerbations or response to inhaled corticosteroids was not demonstrated (33). Furthermore, regardless of the cutoff, COPD patients

\section{REFERENCES}

1. Singh $\mathrm{D}$, Kolsum $\mathrm{U}$, Brightling $C E$, Locantore $\mathrm{N}$, Agusti $\mathrm{A}$, Tal-Singer R; ECLIPSE investigators (2014) Eosinophilic inflammation in COPD: prevalence and clinical characteristics. Eur Respir J. 44(6): 1697-700.

2. George L and Brightling CE (2016) Eosinophilic airway inflammation: role in asthma and chronic obstructive pulmonary disease. Ther Adv Chronic Dis. 7(1): 34-51.

3. Bafadhel M, Peterson S, De Blas MA, Calverley PM, Rennard SI, Richter K, Fagerås M (2018) Predictors of exacerbation risk and response to budesonide in patients with chronic obstructive pulmonary disease: a post-hoc analysis of three randomised trials. The Lancet Respir Med. 6(2): 117-26.

4. Roche N, Chapman KR, Vogelmeier CF, Herth FJF, Thach C, Fogel R, Olsson P, Patalano F, Banerji D, Wedzicha JA (2017) Blood Eosinophils and Response to Maintenance Chronic Obstructive with elevated bEo exhibited no specific characteristic in terms of symptoms, lung function, exacerbation rate, and prognosis (34). Nevertheless, our study confirmed that COPD patients with higher both sEo and bEo during exacerbation showed higher number of exacerbations, but less frequent necessity for hospitalisation due to exacerbation, and shorter hospitalisation in comparison to patients lower sEo and bEo (Table I).

At the same time, results from studies investigating bEo and sEo correlation in COPD patients are also inconsistent. Although relationship between sEo and bEo has been found in COPD (35), the association is still generally weak (Bafedhel 2011). sEo could be a better biomarker than high concentrations of bEo to identify a patient subgroup with more severe COPD, more frequent exacerbations, and increased emphysema by quantitative computed tomography (36). In our study, bEo correlated to sEo (graph 1) and relative peripheral blood eosinophilia predicted sputum eosinophilia at a threshold of $\geq 4 \%$ with specificity of $83.56 \%$ and sensitivity of $93.83 \%$ (graph 2). Similar results were published by Negewo and collaborates who found that bEo correlated with both the percentage and number of sEo and absolute bEo was predictive of sEo. In their study, specificity and sensitivity were $76 \%$ and $60 \%$, respectively at a bEo threshold of $\geq 0.3 \times 109 / \mathrm{L}$. The bEo threshold of $\geq 0.4 \times 109 / \mathrm{L}$ had similar classifying ability but better specificity (91.7\%) (10).

Our study has some advantages and disadvantages. The advantages are: number of patients in groups is satisfactory, groups are homogenous (group C) and there was no droupout of patients. The disadvantage is that the COPD comorbidity with asthma with not excluded, which is otherwise quite demanding and sometimes even impossible in a specific moment. So the results could be applicated on GOLD group C of COPD only.

In conclusion, during COPD exacerbation, relative bEo of $\geq 4 \%$ might identify sputum eosinophilia and indicate better outcome of COPD exacerbation. Further investigations are needed to predict eosinophilic exacerbation in COPD patients, with prior absence of sputum or blood eosinophilia.

Pulmonary Disease Treatment. Data from the FLAME Trial. Am J Respir Crit Care Med. 195(9): 1189-97.

5. Chapman KR, Hurst JR, Frent SM, Larbig M, Fogel R, Guerin T, Banerji D, Patalano F, Goyal P, Pfister P, Kostikas K, Wedzicha JA (2018) Long-term Triple Therapy De-escalation to Indacaterol/ Glycopyrronium in COPD Patients (SUNSET): a Randomized, Double-Blind, Triple-Dummy Clinical Trial. Am J Respir Crit Care Med. 198(3): 329-39.

6. Bafadhel M, McKenna S, Terry S, Mistry V, Reid C, Haldar P, McCormick M, Haldar K, Kebadze T, Duvoix A, Lindblad K, Patel $H$, Rugman $P$, Dodson $P$, Jenkins $M$, Saunders $M$, Newbold $P$, Green RH, Venge P, Lomas DA, Barer MR, Johnston SL, Pavord ID, Brightling CE (2011) Acute exacerbations of chronic obstructive pulmonary disease: identification of biologic clusters and their biomarkers. Am J Respir Crit Care Med. 184(6): 662-71. 
7. Balzano G, Stefanelli F, lorio C, De Felice A, Melillo EM, Martucci M, Melillo G (1999) Eosinophilic inflammation in stable chronic obstructive pulmonary disease. Relationship with neutrophils and airway function. Am J Respir Crit Care Med. 160(5 Pt 1): 1486-92.

8. McDonald VM, Higgins I, Wood LG, Gibson PG (2013) Multidimensional assessment and tailored interventions for COPD: respiratory utopia or common sense? Thorax. 68(7): 691-4.

9. Leigh R, Pizzichini MM, Morris MM, Maltais F, Hargreave FE, Pizzichini E (2006) Stable COPD: predicting beneft from highdose inhaled corticosteroid treatment. Eur Respir J. 27(5): 96471.

10. Negewo NA, McDonald VM, Baines KJ, Wark PA, Simpson JL, Jones PW, Gibson PG (2016) Peripheral blood eosinophils: a surrogate marker for airway eosinophilia in stable COPD. Int J Chron Obstruct Pulmon Dis. 11: 1495-504.

11. Pavord ID, Bafadhel M (2013) Exhaled nitric oxide and blood eosinophilia: independent markers of preventable risk. J Allergy Clin Immunol. 132(4): 828-9.

12. Baines KJ, Pavord ID, Gibson PG (2014) The role of biomarkers in the management of airways disease. Int J Tuberc Lung Dis. 18(11): 1264-68

13. Landis SH, Suruki R, Hilton E, Compton C, Galwey NW (2017) Stability of Blood Eosinophil Count in Patients with COPD in the UK Clinical Practice Research Datalink. COPD. 14(4): 382-8.

14. Westerhof GA, Korevaar DA, Amelink M, de Nijs SB, de Groot JC, Wang J, Weersink EJ, ten Brinke A, Bossuyt PM, Bel EH (2015) Biomarkers to identify sputum eosinophilia in different adult asthma phenotypes. Eur Respir J. 46(3): 688-96.

15. Eltboli O, Mistry V, Barker B, Brightling CE (2015) Relationship between blood and bronchial submucosal eosinophilia and reticular basement membrane thickening in chronic obstructive pulmonary disease. Respirology. 20(4): 667-70.

16. Global Initiative for Chronic Obstructive Lung Disease (GOLD) 2019 report; available at: https://goldcopd.org/wp-content/ uploads/2018/11/GOLD-2019-v1.7-FINAL-14Nov2018-WMS pdf Last updated: November 14th, 2018.

17. Rajala $K$, Lehto JT, Sutinen $E$, Kautiainen $H$, Myllärniemi $M$, Saarto T (2017) mMRC dyspnoea scale indicates impaired quality of life and increased pain in patients with idiopathic pulmonary fibrosis. ERJ Open Res. 3(4). pii: 00084-2017.

18. Houben-Wilke S, Janssen DJA, Franssen FME, Vanfleteren LEGW, Wouters EFM, Spruit MA (2018) Contribution of individual COPD assessment test (CAT) items to CAT total score and effects of pulmonary rehabilitation on CAT scores. Health Qual Life Outcomes. 16(1): 205.

19. Marcos PJ, Sanjuán $P$, Huerta A, Nieto-Codesido I, FerreiraGonzalez L, Sibila O, Restrepo MI (2017) Relationship Between Severity Classification of Acute Exacerbation of Chronic Obstructive Pulmonary Disease and Clinical Outcomes in Hospitalized Patients. Cureus. 9(1): e988.

20. Wedzicha JA (2016) Eosinophils as Biomarkers of Chronic Obstructive Pulmonary Disease Exacerbation Risk. Maybe Just for Some? Am J Respir Crit Care Med. 193(9): 937-8.

21. Vedel-Krogh S, Nielsen SF, Lange $P$, Vestbo J, Nordestgaard BG (2016) Blood eosinophils and exacerbations in chronic obstructive pulmonary disease. The Copenhagen General Population Study. Am J Respir Crit Care Med. 193(9): 965-74.

22. 2Donaldson GC, Law M, Kowlessar B, Singh R, Brill SE, Allinson JP, Wedzicha JA (2015) Impact of prolonged exacerbation recovery in chronic obstructive pulmonary disease. Am J Respir Crit Care Med. 192: 943-50.
23. Pavord ID, Lettis S, Locantore N, Pascoe S, Jones PW, Wedzicha JA Barnes N (2016) Blood eosinophils and inhaled corticosteroid/ long-acting $\beta$-2 agonist efficacy in COPD. Thorax. 71(2): 118-25.

24. Couillard S, Larivée P, Courteau J, Vanasse A (2017) Eosinophils in COPD Exacerbations Are Associated With Increased Readmissions. Chest. 151(2): 366-73.

25. Barnes NC, Sharma R, Lettis S, Calverley PM (2016) Blood eosinophils as a marker of response to inhaled corticosteroids in COPD. Eur Respir J. 47(5): 1374-82.

26. Price D, Rigazio A, Postma D (2014) Blood eosinophilia and the number of exacerbations in COPD patients [abstract]. Eur Respir J. 44(Suppl 58): 4416.

27. Pascoe S, Locantore N, Dransfeld MT, Barnes NC, Pavord ID (2015) Blood eosinophil counts, exacerbations, and response to the addition of inhaled fluticasone furoate to vilanterol in patients with chronic obstructive pulmonary disease: a secondary analysis of data from two parallel randomised controlled trials. Lancet Respir Med. 3(6): 435-42.

28. Siddiqui SH, Guasconi A, Vestbo J, Jones $P$, Agusti A, Paggiaro $P_{\text {, }}$ Wedzicha JA, Singh D (2015) Blood eosinophils: a biomarker of response to extrafine beclomethasone/formoterol in chronic obstructive pulmonary disease. Am J Respir Crit Care Med. 192(4): 523-5.

29. Siddiqui SH, Pavord ID, Barnes NC, Guasconi A, Lettis S, Pascoe S, Petruzzelli S (2018) Blood eosinophils: a biomarker of COPD exacerbation reduction with inhaled corticosteroids. Int $J$ Chron Obstruct Pulmon Dis. 13: 3669-76.

30. Watz $H$, Tetzlaff $K$, Wouters EF, Kirsten A, Magnussen $H_{\text {, }}$ Rodriguez-Roisin R, Vogelmeier C, Fabbri LM, Chanez P, Dahl R, Disse B, Finnigan H, Calverley PM (2016) Blood eosinophil count and exacerbations in severe chronic obstructive pulmonary disease after withdrawal of inhaled corticosteroids: a post-hoc analysis of the WISDOM trial. Lancet Respir Med. 4(5): 390-8.

31. Bélanger M, Couillard S, Courteau J, Larivée P, Poder TG, Carrier N, Girard K, Vézina FA, Vanasse A (2018) Eosinophil counts in first COPD hospitalizations: a comparison of health service utilization. Int J Chron Obstruct Pulmon Dis. 13: 3045-54.

32. Ho J, He W, Chan MTV, Tse G, Liu T, Wong SH, Leung CCH, Wong WT, Tsang S, Zhang L, Chan RYP, Gin T, Leung J, Lau BWM, Wu WKK, Ngai SPC (2017) Eosinophilia and clinical outcome of chronic obstructive pulmonary disease: a meta-analysis. Sci Rep. 7(1): 13451

33. Adir $\mathrm{Y}$, Hakrush $\mathrm{O}$, Shteinberg $\mathrm{M}$, Schneer $\mathrm{S}$, Agusti $A$ (2018) Circulating eosinophil levels do not predict severe exacerbations in COPD: a retrospective study. ERJ Open Res. 4(3). pii: 00022-2018.

34. Zysman M, Deslee G, Caillaud D, Chanez P, Escamilla R, CourtFortune I, Nesme-Meyer P, Perez T, Paillasseur JL, Pinet C, Jebrak G, Roche N, Burgel PR (2017) Relationship between blood eosinophils, clinical characteristics, and mortality in patients with COPD. Int J Chron Obstruct Pulmon Dis. 12: 1819-24.

35. Hartjes FJ, Vonk JM, Faiz A, Hiemstra PS, Lapperre TS, Kerstjens HAM, Postma DS, van den Berge M; and the Groningen and Leiden Universities Corticosteroids in Obstructive Lung Disease (GLUCOLD) Study Group (2018) Predictive value of eosinophils and neutrophils on clinical effects of ICS in COPD. Respirology. 23(11): 1023-31.

36. Hastie AT, Martinez FJ, Curtis JL, Doerschuk CM, Hansel NN, Christenson S, Putcha N, Ortega VE, Li X, Barr RG, Carretta EE, Couper DJ, Cooper CB, Hoffman EA, Kanner RE, Kleerup E, O'Neal WK, Paine R 3rd, Peters SP, Alexis NE, Woodruff PG, Han MK, Meyers DA, Bleecker ER; SPIROMICS investigators. (2017) Association of sputum and blood eosinophil concentrations with clinical measures of COPD severity: an analysis of the SPIROMICS cohort. Lancet Respir Med. 5(12): 956-67. 